\title{
SERVICIOS COMERCIALES EN LA INDUSTRIA DEL ASERRÍO DE LA REGIÓN DEL MAULE, CHILE
}

\author{
Mauricio Ponce D. ${ }^{1}$, Eduvijis Valenzuela B.
}

\section{RESUMEN}

El marketing de productos forestales es el área de la empresa menos asumida por los productores madereros. Ellos trabajan para mejorar sus sistemas de producción, con tecnología antigua y enfrentan importantes restricciones de abastecimiento, tanto en cantidad como en calidad. En este contexto, se realizó un estudio exploratorio en 18 aserraderos de la Región del Maule $(22,5 \%$ del total) para diagnosticar la existencia de servicios comerciales en estas empresas.

El 89\% de los encuestados declara tener los servicios de almacenamiento, rotulación, embalaje e industrialización, con amplios niveles de implementación. Respecto de otros servicios como entrega oportuna, atención de cliente, solución de problemas, garantía del producto, reclamos y crédito, son los grandes y medianos los que destacan (48\%), los pequeños no cuentan de forma adecuada con estos servicios para sus clientes. A pesar de los resultados obtenidos, aún no asumen los servicios comerciales como parte de una estrategia empresarial, que dé utilidad a los consumidores, aspectos que deben ser desarrollados para lograr una etapa de consolidación de sus empresas en los mercados locales e internacionales.

Palabras claves: Marketing forestal, servicios al consumidor, aserraderos.

\section{SUMMARY}

The forest products' marketing is the more diminished business area of sawmills industrials. They work to improve their production systems with old technology and must encourage mains constraint likes the supply, both in quantity and quality. In this context, a exploratory survey did a diagnostic about the commercial services in sawmills of Maule Region (22.5\% of all).

The $89 \%$ of polled declared to have storage, labelling, packing and industrialization services, with wide levels of implementation. Respect to the other services likes a delivery, client's attention, problems solution, guaranty products, claims and credit service, the big and medium sawmills stand out (48\%), and the small one haven't these services for their customers adequately. In spite of results, the sawmills do not assume the commercial services yet, as part of a management strategy, which give utility to the clients, those aspects must be developed to achieve a consolidation stage at companies at local and international markets.

Keywords: Forest marketing, customer services, sawmills.

1 Universidad de Talca, Facultad de Ciencias Forestales. Talca. Chile. mponce@utalca.cl 


\section{INTRODUCCIÓN}

La comercialización de productos involucra los procesos que van desde la obtención de la materia prima hasta que estos llegan a manos del consumidor o cliente; incluye transporte, clasificación, control de calidad, mantención de inventarios y otros, a fin de satisfacer sus necesidades (FAO, 1997). El desarrollo técnico, social y económico de los últimos años, ubican la comercialización en el centro de las actividades de una empresa, orientando el producto o servicio dentro de un sistema comercial, en momento y lugar requeridos, con la máxima utilidad posible, registrándolo en la conducta de los consumidores, orientándolos en su decisión de compra y llevándoles a ser agentes de divulgación de productos, marcas y empresas. Un buen producto no asegura el éxito de un negocio, por esto es indispensable combinar elaboración con estrategias de comercialización.

La importancia de la comercialización en las actividades forestales, en el nivel industrial y comercial, se expresa en la demanda, las estructuras demográficas, el desarrollo económico y los aspectos sociales y ambientales, mientras que para el productor aparecen grandes cambios en el abastecimiento de materias primas y la aparición de nuevos productos (Juslin y Lintu, 1997). Se prevé asimismo que se generarán demandas específicas en base al consumo de productos certificados, obtenidos mediante un manejo sustentable de los bosques, que crearán nichos de mercado específicos. Además, los consumidores tendrán mayor ingerencia en las decisiones del sector, influyendo en el desarrollo de productos, en un mercado cada vez más competitivo.

En la Región del Maule existen 151 aserraderos entre móviles y permanentes, representando el 99\% de la industria primaria, con una participación del 17,1\% de la producción nacional (INFOR, 2009b). Sin embargo, según González y Carrasco (2003), es aún difícil diferenciar la industria primaria de la secundaria. Talca es la provincia de la región que más aserraderos concentra, con 92 unidades. Estos aserraderos poseen características productivas bien definidas de acuerdo al estudio de Del Solar et al. (2008), por ello las estrategias de servicios comerciales que desarrollan resultan relevantes para su desempeño, ya que influirán en las decisiones de los consumidores respecto de dónde y cómo comprar.

Según Cáceres et al. (1987), la distribución de productos industriales usa canales más cortos o directos, controlando así la distribución con estabilidad de las operaciones y servicios comerciales, de acuerdo a las capacidades de la empresa. Por su parte, el consumidor demanda estos servicios que redunden en nuevas aplicaciones, acceso y tratamiento de la información y logren responder a una o más necesidades (Verdugo et al., 2007).

Los servicios comerciales corresponden a acciones, procesos y ejecuciones que forman parte de la oferta de bienes manufacturados (Zaithaml y Bitner, 2002), sus características son intangibilidad, heterogeneidad, inseparabilidad y ausencia de propiedad. Caldentey (1992) identifica los siguientes en el ámbito silvoagropecuario: Transporte, almacenamiento, industrialización, embalaje, rotulación y entrega oportuna.

En el sector estudiado, Moguillansky y Silva (2001) identificaron dos grandes problemas, la comercialización y la búsqueda de mercados para productos con mayor valor agregado. En general, los aspectos relacionados con el desarrollo de mercados son aún temas pendientes 
en el área productiva forestal madera en varios países, según lo señalado por Jenkins y Smith (1999) ratificado por Juslin y Hansen (2003); otro aspecto que también la define es la cantidad y característica de la fuerza laboral, y su reducido impacto en la productividad (Riffo, 2007). INFOR (2009a) releva variables clave en la distribución de madera aserrada, que ordenadas jerárquicamente resultan en: Tiempo de reacción, atención al cliente, capacitación de la fuerza de ventas, valor agregado, relación entre el cliente y el proveedor, servicio pos venta, relación precio calidad y certificación. Por otro lado, Ponce et al. (2010) señalan entre las principales características productivas de este sector, en la región de referencia, la escasa orientación a desarrollar nuevos mercados, escaso volumen y calidad del mix de productos ofrecidos, escaso uso de las tecnologías de información y comunicación y fuerte competencia con el retail en estrategias de precio y promoción.

El objetivo de este estudio fue realizar un diagnóstico exploratorio de los servicios comerciales que la industria de transformación primaria de la madera de la Región del Maule ofrece a sus clientes, y proponer acciones que propendan a un mejor desempeño de estos servicios.

\section{METODOLOGÍA}

Para el estudio se utilizó una muestra de 18 empresas de aserrío (transformación primaria), ubicadas en la Región del Maule, de las provincias de Talca (14 empresas; 2 grandes, 11 medianas y 1 pequeña), Cauquenes (3 empresas; 2 medianas y 1 pequeña) y Linares (1 empresa mediana). Como criterio de tamaño se usó el rango de producción utilizado por INFOR (2009a). El catastro se elaboró a partir de datos de la Corporación Nacional Forestal (CONAF, 2007) e INFOR (2009b). Los criterios de selección fueron: a) Productores de madera aserrada y dimensionada como productos principales, b) Materia prima principal Pinus radiata (D. Don) y c) Aserraderos permanentes. Se identificaron 80 aserraderos de este tipo, con lo cual la cobertura del estudio alcanzó el 22,5\%.

Se elaboró una encuesta con preguntas cerradas y de seguimiento, la que fue ajustada en dos instancias piloto. Su aplicación se llevó a cabo ente los meses de febrero y abril del año 2009. El instrumento constó de los siguientes ítems: Descripción general de la empresa; identificación de productos; características de la materia prima, del servicio de venta y los servicios comerciales. Los servicios comerciales considerados en el estudio fueron: Almacenamiento, transporte, rotulación, embalaje, industrialización, entrega oportuna, garantía del producto, sistemas de pago y atención al cliente. Los resultados fueron tabulados usando planilla Excel.

\section{RESULTADOS Y DISCUSIÓN}

El principal mix de productos que los aserraderos encuestados elaboran son madera verde, madera seca y madera cepillada en diferentes dimensiones, en función de pedidos y características de la materia prima utilizada. Un 38\% señala que trabaja bajo normas de calidad, especialmente ISO 9000.

En relación a las principales características de la materia prima (rollizo) que buscan los productores, se encuentran la ausencia de ataque de insectos y hongos, diámetros adecuados 
y conicidad como los más importantes; le siguen la adquisición de rollizos con manejo, ausencia de arqueaduras y largos de 3,2 m; en menor importancia están el contenido de humedad y calidad de la corteza. En el contexto, un $50 \%$ de los encuestados expresa que lo más importante es obtener mayor aprovechamiento de las trozas, un 17\% producir bajo normas de calidad y un $80 \%$ los aserraderos medianos y grandes señalan agregar valor al producto, aunque no se identifica cabal claridad en el uso de este concepto. Cabe indicar que para el $61 \%$ de los entrevistados, los proveedores de materias primas son las grandes empresas forestales del país y para el $22 \%$ es una mezcla de estas con medianos proveedores, aspecto que es reconocido como una de las grandes restricciones en el desarrollo del sector pyme (Ponce et al., 2010).

En relación a la forma cómo realizan la venta de sus productos, el 50\% del total señala hacerlo directamente, todos medianos y pequeños productores; un $11 \%$ lo hace únicamente en Santiago, correspondiente a uno mediano; otro 11\% lo concreta en ambos puntos; el resto lo hace directamente en el extranjero o bien usando una combinación de oficina en Santiago, correspondiendo a grandes empresas. En cuanto a la comercialización de los productos, el $93 \%$ de los grandes y medianos aserraderos declara tener personal destinado para ello; sin embargo, en el trabajo hecho por Ponce et al. (2010) se observó que esta actividad es realizada por el dueño en los medianos aserraderos, o un encargado del área de producción, pero claramente no cuentan con las competencias requeridas para hacerlo. En las pequeñas empresas no hay alguien definido para esta actividad, sí es posible identificar que las grandes empresas cuentan con personal preparado para esta acción.

Un $64 \%$ de los medianos y el total de los grandes aserraderos, declaran hacer uso de recursos económicos para publicidad; sin embargo, se desconoce el monto y el destino de éstos, incluso si el concepto de publicidad es comprendido a cabalidad y no pasa de ser solo la colocación un cartel carretero.

Los siguientes cuadros muestran datos agregados (en porcentaje) de los servicios declarados que poseen las empresas destinados a sus clientes, considerando los 18 aserraderos encuestados de la muestra.

\section{Cuadro $\mathrm{N}^{\circ} 1$}

\section{SERVICIOS COMERCIALES PRESENTES EN LA INDUSTRIA PRIMARIA} (Porcentaje)

\begin{tabular}{|c|c|c|c|c|}
\hline Tipo Empresa & Almacenamiento & Rotulación & Embalaje & Industrialización \\
\hline Grande & 11 & 11 & 11 & 11 \\
Mediana & 72 & 72 & 72 & 78 \\
Pequeña & 6 & 11 & 6 & 11 \\
\hline Total & $\mathbf{8 9}$ & $\mathbf{9 4}$ & $\mathbf{8 9}$ & $\mathbf{1 0 0}$ \\
\hline
\end{tabular}




\section{Cuadro $\mathrm{N}^{\circ} 2$ \\ OTROS SERVICIOS COMERCIALES EVALUADOS \\ (Porcentaje)}

\begin{tabular}{|c|c|c|c|c|c|c|}
\hline \multirow{2}{*}{$\begin{array}{c}\text { Tipo } \\
\text { Empresa }\end{array}$} & \multicolumn{6}{|c|}{ Otros Servicios } \\
\cline { 2 - 7 } & $\begin{array}{c}\text { Entrega } \\
\text { Oportuna }\end{array}$ & $\begin{array}{c}\text { Atención } \\
\text { Cliente }\end{array}$ & $\begin{array}{c}\text { Solución } \\
\text { Problemas }\end{array}$ & $\begin{array}{c}\text { Garantía } \\
\text { Producto }\end{array}$ & $\begin{array}{c}\text { Posibilidad } \\
\text { Reclamos }\end{array}$ & $\begin{array}{c}\text { Existencia } \\
\text { Crédito }\end{array}$ \\
\hline Grande & 6 & 11 & 11 & 11 & 11 & 11 \\
Mediana & 72 & 78 & 78 & 36 & 67 & 33 \\
Pequeña & 11 & 6 & 6 & 0 & 6 & 0 \\
\hline Total & $\mathbf{8 9}$ & $\mathbf{9 4}$ & $\mathbf{9 4}$ & $\mathbf{3 9}$ & $\mathbf{8 3}$ & $\mathbf{4 4}$ \\
\hline
\end{tabular}

En cuanto a las grandes empresas, se puede señalar que son las que poseen todos los servicios comerciales evaluados; el almacenamiento lo realizan en bodegas o galpones especialmente acondicionado para ello, reconociendo que esto permite proteger los productos, no señalándose que sirva, por ejemplo, para mantener las fluctuaciones del stock. Respecto a la mantención de un sistema de transporte, tanto terrestre como marítimo, indican que les permite puntualidad y protección al producto. En cuanto a la rotulación, sólo permitiría la identificación de la empresa, que se aplicaría más por exigencia de la empresa a la cual se destina el producto o bien por cumplir una normativa. En relación al embalaje, se reconoce como algo importante y que se realiza más que nada por la presentación del producto. Al servicio de industrialización se le reconoce como la posibilidad de agregar valor agregado y mayor variedad de productos, aspecto que se destaca, pero del cual tienen menor holgura para cambiar el conjunto de productos en elaboración, si se les compara con un aserradero de tamaño mediano.

En cuanto a los servicios de venta, destacan el cumplimiento en fechas de entrega, existencia de unidades de ventas y capacidad para resolver los problemas de los clientes de "buena forma" y de manera inmediata. La explicación a la existencia de garantía del producto se basa en que éstos son elaborados bajo normas y los sistemas de reclamo son a través del teléfono o encuestas. El sistema de pago preferentemente utilizado es el crédito, que permite un adecuado flujo de caja y opciones de negociación con los clientes, cuyo contacto es preferentemente a través del correo electrónico y el teléfono.

Las empresas de mediano tamaño poseen capacidad de almacenamiento, aunque no todas son cubiertas, el $71 \%$ señala que se posee para proteger los productos, mientras que un $14 \%$ indican que es para disponibilidad de stock e igual porcentaje no lo considera importante. En cuanto al transporte por tierra, el $71 \%$ tiene este servicio que se realiza utilizando camiones; su importancia radica en hacer llegar el producto a los clientes, con puntualidad y seguridad en el abastecimiento. Un 93\% rotula sus productos, el 50\% poniendo el nombre de otra empresa y el resto a fin de cumplir con la norma NIMF 15; sin embargo, el 55\% reconoce la importancia de la rotulación para identificar su empresa. En cuanto al uso de embalaje, el $43 \%$ lo asume como orden superior de la empresa, mientras que un $50 \%$ lo considera parte de la presentación del producto. Estos productores ven en el servicio de industrialización como una forma de 
supervivencia y de añadir valor a los productos, lo que permite observar que son un grupo con mayor adaptación a los cambios de productos que se demandan.

Por otro lado, un 93\% considera que la entrega oportuna es la forma de cumplir y mantener los clientes. En el mismo sentido, todos valoran la atención al cliente, solucionar sus problemas, dar garantía al producto y aceptar reclamos, aunque no se advierte un manejo cabal de lo que estos aspectos involucran. En relación a los sistemas de pagos, predomina el contado por sobre el crédito, que aún siendo aceptado, solo uno señala que es habitual. El 93\% indica que su sistema pago permite un flujo de caja para "mover el negocio". Respecto de cómo se establecen los pedidos, el $71 \%$ utiliza la orden de compra, el $14 \%$ el teléfono y ninguno la Internet.

En relación a las pequeñas empresas, el almacenamiento de productos se realiza tanto bajo techo como al aire libre, aunque todos reconocen que es necesario protegerlos. No poseen capacidad de transporte propio como parte de la oferta, reconociendo que tenerlo permitiría asegurar que el producto llegue al cliente. En relación a la rotulación, se señala que ello sirve para poner la marca de la empresa que comercializa la madera e identificar la que se produce bajo la norma NIMF 15; sólo uno reconoce que este servicio puede ayudar a la promoción de la empresa. Por otro lado, se identifica el uso de embalaje del producto sólo por la presentación. Estos productores reconocen en el servicio de industrialización la posibilidad de agregar mayor valor a los productos elaborados. En cuanto a la entrega oportuna, señalan que permite cumplir con la fecha; mientras que el $50 \%$ dice tener atención al cliente y que soluciona los problemas con éste de "buena forma". La garantía del producto sólo se otorgaría si se daña y que los reclamos se hacen vía telefónica. Para el cobro de sus productos utilizan el pago contado de preferencia, aunque también otorgan crédito, haciendo referencia al mejoramiento del flujo de caja como criterio para su aplicación. Llama la atención en este segmento que declara usar el correo electrónico para la realización de contratos con los clientes.

\section{CONCLUSIONES}

En términos generales, la venta de los productos se realiza en forma directa, encontrándose escaso personal profesional en medianos y pequeños aserraderos que tengan estas competencias.

En cuanto a sus principales requerimientos de materia prima, son sanidad y forma, seguido de los diámetros y presencia de nudos. Las que presentan mayor indiferencia son la calidad de la corteza y el contenido de humedad.

Las empresas madereras medianas y pequeñas no tienen seguimiento de sus productos, ni utilizan sellos que distingan su proveniencia, pero sí colocan marcas de terceros.

Los grandes aserraderos poseen servicios de transporte propio y hacen uso de embalaje para la presentación de sus productos. Aunque tienen un buen mix de productos, no tienen la plasticidad suficiente para adaptar su producción a nuevas peticiones de los clientes.

Los medianos aserraderos aplican todos los servicios, pero ocurre principalmente por una petición de los clientes, más que por una acción que busque dar mayor valor al producto. 
Destaca su capacidad de adaptarse a nuevas exigencias de los clientes en cuanto a productos y escasamente en servicios, encontrándose en una etapa muy rudimentaria en algunos casos.

Los servicios comerciales en pequeños productores están presentes en menor cantidad, las que se asocian a sus capacidades financieras y a los requerimientos de los clientes, que son menos complejas. Por lo general trabajan para otras empresas comercializadoras y productoras.

Como recomendaciones para el desarrollo del sector comercial de las empresas estudiadas, especialmente medianas, están el desarrollar la capacidad de tener un abastecimiento sostenido en cantidad y calidad, que permita mejorar la utilidad del consumidor de los productos que vende; profesionalizar las diferentes áreas de la industria generando las competencias necesarias a diferentes niveles de preparación, especialmente en el área comercial, que los lleve a ampliar y profundizar el conocimiento tanto del mercado local como nacional. Además, deben incorporar normas de producción y calidad; elaborar estrategias de marketing básico en torno a precios, promoción y distribución. Finalmente, requieren aprender a manejar aspectos financieros y relación con la banca, que le permitan acceder a mejores condiciones de financiamiento de corto y largo plazo.

\section{REFERENCIAS}

Cáceres, J., Briz, J., Rebollo, A. y Muñoz, P., 1987. La economía de la distribución comercial. Editorial Ariel, España. 307p.

Caldentey, P., 1992. Comercialización de productos agrarios. Editorial Agrícola Española s.a. $279 \mathrm{p}$.

CONAF, 2007. Directorio de empresas forestales 2007 Región del Maule. 16 p. Corporación Nacional Forestal, Chile

Del Solar, R., Chacón, I. y Ponce, M., 2008. Plan agregado de producción en barracas madereras. Estudio de caso para una pequeña industria. Maderas, Ciencia y Tecnología 10(2): 77-92.

FAO, 1997. Provisional outlook for global forest products consumption, production and trade to 2010. Food and Agricultural Organization, Italia.

González, C. y Carrasco, L., 2003. Diagnóstico de la demanda de madera de productos madereros en la Región del Maule. Talca. Documento de la CONAF. 73 p.

INFOR, 2009a. Importancia de la distribución comercial. En resúmenes del seminario: Rueda de negocios forestal. FITAL 2009. Instituto Forestal, Chile

INFOR, 2009b. Industria del aserrío 2009. Santiago, Chile. Consultado el 03.06.2009. Disponible en http://www.infor.cl Instituto Forestal, Chile

Jenkins, M. B. and Smith, E.T., 1999. The business of sustainable forestry. Strategies for an 
industry in transition. Chapter 3. Island Press.

Juslin, H, y Lintu, L., 1997. Respuesta a los cambios en la oferta y la demanda de productos forestales a través de la mejora del marketing. Congreso Forestal Mundial Antalya, Turquía. Consultado 16 de junio 2008. Disponible en: http://fao.org/forestry/docrep/wfcxi/ publi/V4/ T212S/2-1.htm

Juslin, H. and Hansen, E., 2003. Strategic marketing in the global forest industries. Authors Academics Press. P. $417-552$.

Moguillasky, G. y Silva, V., 2001. Estrategias empresariales y políticas públicas: el futuro del complejo forestal en Chile. En: Más allá del bosque: transformar el modelo exportador. FLACSO. p. $107-144$.

Ponce, M., Carrasco, L., Maureira, D. y Rojas, N., 2010. La empresa PyME forestal maderera de la región del Maule. Propuesta de un modelo de negocio. Programa de Difusión y Transferencia Tecnológica para contribuir a mejorar la competitividad de las MIPYMES forestales de la Región del Maule. Informe Final. 16 p.

Riffo, L., 2007. Análisis económico productivo y fomento a la investigación científica y tecnológica y a la innovación. Curso ILPES/AUR. P. 7 - 45.

Verdugo, C., Muñoz, A., Toledo, B. y Cáceres, R., 2007. El transporte como hecho gravado por el impuesto a las ventas y servicios. Memoria de pregrado de Derecho. Universidad Pedro de Valdivia. Escuela de Derecho. 80 p.

Zeithaml, V. y Bitner, M., 2002. Introducción a los servicios. In: Marketing de servicios: un enfoque de integración del cliente a la empresa. México. p. 1 - 35. 